Nadine Meertens - Michael H. Stoffel · Pascal Cherpillod Riccardo Wittek · Marc Vandevelde · Andreas Zurbriggen

\title{
Mechanism of reduction of virus release and cell-cell fusion in persistent canine distemper virus infection
}

Received: 19 March 2003 / Revised: 14 May 2003 / Accepted: 14 May 2003 / Published online: 21 June 2003

(C) Springer-Verlag 2003

\begin{abstract}
Canine distemper virus (CDV), a mobillivirus related to measles virus causes a chronic progressive demyelinating disease, associated with persistence of the virus in the central nervous system (CNS). CNS persistence of morbilliviruses has been associated with cell-to-cell spread, thereby limiting immune detection. The mechanism of cell-to-cell spread remains uncertain. In the present study we studied viral spread comparing a cytolytic (non-persistent) and a persistent CDV strain in cell cultures. Cytolytic CDV spread in a compact concentric manner with extensive cell fusion and destruction of the monolayer. Persistent CDV exhibited a heterogeneous cell-tocell pattern of spread without cell fusion and 100-fold reduction of infectious viral titers in supernatants as compared to the cytolytic strain. Ultrastructurally, low infectious titers correlated with limited budding of persistent $\mathrm{CDV}$ as compared to the cytolytic strain, which shed large numbers of viral particles. The pattern of heterogeneous cell-to-cell viral spread can be explained by low production of infectious viral particles in only few areas of the cell membrane. In this way persistent CDV only spreads to a small proportion of the cells surrounding an infected one. Our studies suggest that both cell-to-cell spread and limited production of infectious virus are related to reduced expression of fusogenic complexes in the cell membrane. Such complexes consist of a synergistic configuration of the attachment $(\mathrm{H})$ and fusion $(\mathrm{F})$ proteins on the cell surface. $\mathrm{F}$ und $\mathrm{H}$ proteins exhibited a marked degree of colocalization in cytolytic CDV infection but not in persistent
\end{abstract}

N. Meertens · P. Cherpillod $\cdot$ M. Vandevelde $\cdot$ A. Zurbriggen ( $\bowtie)$ Department of Clinical Veterinary Medicine,

Division of Clinical Research, University of Bern,

3012 Bern, Switzerland

Tel.: +41-31-6312509, Fax: +41-31-6312538

M. H. Stoffel

Institute of Veterinary Anatomy, University of Bern,

3012 Bern, Switzerland

R. Wittek

University of Lausanne, 1015 Lausanne, Switzerland
CDV as seen by confocal laser microscopy. In addition, analysis of CDV F protein expression using vaccinia constructs of both strains revealed an additional large fraction of uncleaved fusion protein in the persistent strain. This suggests that the paucity of active fusion complexes is due to restricted intracellular processing of the viral fusion protein.

Keywords Canine distemper virus · Virus spread · Persistence

\section{Introduction}

Canine distemper virus (CDV), a morbillivirus, closely related to measles virus (MV), induces a multifocal demyelinating disease in dogs similar to multiple sclerosis (MS) in man [1]. Initial demyelination is associated with viral replication in the white matter of the central nervous system (CNS). The antiviral immune response, with invasion of immune cells in the CNS, leads to viral clearance within the inflammatory lesions. However, despite an apparently effective immune response, CDV can persist outside of the lesions and continues to replicate and spread to other areas [23]. As a result of viral persistence, a chronic progressive and relapsing disease develops.

The mechanism of persistence of morbilliviruses is not well understood. Defective MVs, which occur in human subacute sclerosing panencephalitis (SSPE) $[4,16]$, and restricted infection with full transcription but limited translation of $\mathrm{CDV}[13,22]$ are thought to contribute to persistence. An essential feature of persistence of MV appears to be transmission of the virus from one cell to the other $[11,12]$, whereby the cells supporting the infection are not killed. Our previous studies comparing an attenuated non-persistent strain, Onderstepoort (OP)-CDV, with persistent A75/17-CDV in brain cell cultures showed that distemper virus persistence is associated with non-cytolytic cell-to-cell spread in a seemingly selective pattern [23]. Absence of cytolysis in CNS cells in which the virus replicates probably limits activation of antigen presenta- 
tion mechanisms. Selective cell-to-cell spread also tends to limit immune recognition of viral antigen [23]. It is not clear how such cell-to-cell spread of CDV takes place. The distinct morphological impression in CDV infection on immunolabeled preparations in vitro and in vivo is that infected cells are in contact with each other, frequently by their cell processes [23]. This is consistent with the view that nucleocapsids of persistent morbilliviruses may be transported by way of fused cell processes $[6,15]$ or in the case of neurons by way of synapses [10]. In the present study we used titration experiments, electron microscopy and confocal laser microscopy in infected cell cultures to further investigate the mechanism of spread of CDV.

\section{Materials and methods}

Virus

Two strains of CDV were used in this study. A tissue culture-adapted A75/17 strain (A75/17-CDV) was obtained by passaging the virulent A75/17-CDV strain (Gift from M. Appel, Cornell University, Ithaca, NY) 15 times in Vero cells. The resulting virus produces a persistent, noncytolytic infection in primary dog brain cell cultures like the wild-type progenitor as well as in Vero cells [8]. Sequence analysis of both wild type and passaged A75/17-CDV revealed minor structural differences in the $\mathrm{M}, \mathrm{P}$, and L proteins, whereas both surface proteins remained identical (unpublished results). The tissue culture-adapted OP-CDV strain was propagated in Vero cells.

\section{Antibodies}

For the demonstration of viral proteins, we used the following monoclonal antibodies: to the nucleocapsid protein ( $\mathrm{N}$ protein), D110 $[2,8]$; to the matrix $(\mathrm{M})$ protein, XI-6; to the attachment $(\mathrm{H})$ protein, XI-23. In addition, we used rabbit antibodies to the fusion (F) and $\mathrm{H}$ proteins [5]. All of these antibodies recognize their respective proteins of both CDV strains.

\section{Cell lines}

Vero cells were seeded at $3 \times 10^{6} /$ petri dish containing glass coverslips [22] in Dulbecco's Modified Eagle Medium (DMEM) with $5 \%$ fetal calf serum (FCS) and 1\% penicillin-streptomycin (PS).

\section{Infections}

Sub-confluent Vero cell cultures were infected with CDV diluted in DMEM without FCS for $1 \mathrm{~h}$. The cells were infected with $6 \times 10^{4}$ TCID A75/17-CDV/petri dish, and $4 \times 10^{3}$ TCID OP-CDV/petri dish. This resulted in an initial pattern of widely separated single cell infection, which is suited for morphological evaluations. After infection, the medium was replaced with DMEM containing 5\% FCS and $1 \%$ PS.

\section{Trypsin treatment of cell cultures}

A75/17-CDV-infected and corresponding uninfected cells were treated $24 \mathrm{~h}$ post infection (p.i.) with $3 \mu \mathrm{g}$ or $5 \mu \mathrm{g} / \mathrm{ml}$ trypsin (Sigma, Buchs, Switzerland) in PBS without $\mathrm{Ca}^{2+}$ and $\mathrm{Mg}^{2+}$ for $6 \mathrm{~min}$ at $37^{\circ} \mathrm{C}$. The cells were then washed twice with tissue culture medium. At 24 and $48 \mathrm{~h}$ after trypsin treatment, the tissue cultures were fixed and processed for immunohistochemistry using the monoclonal antibody D110. They were screened for syncytia formation by hematoxylin/eosin staining.

\section{Confocal laser microscopy}

After $18 \mathrm{~h}$ for the OP-CDV-infected cells, and $36 \mathrm{~h}$ for the A75/17CDV-infected cells, the cells were fixed with $4 \%$ PBS-buffered paraformaldehyde for $20 \mathrm{~min}$, and washed in TBS for $30 \mathrm{~min}$. The unspecific binding sites were blocked with $5 \%$ normal goat serum in TBS during $30 \mathrm{~min}$. The first antibodies were diluted in TBS as follows : mouse-anti-N protein (D110) 1:1, mouse-anti-H protein (XI-23) undiluted, mouse-anti-M protein (XI-6) undiluted, rabbitanti-F protein $(\mathrm{RaF}) 1: 100$, rabbit-anti-H protein $(\mathrm{RaH}) 1: 100$. Incubation was performed at $37^{\circ} \mathrm{C}$ for $2 \mathrm{~h}$. The cells were washed for $15 \mathrm{~min}$. The fluorescent label-coupled second antibody (goat antimouse-FITC or goat-anti-rabbit-rhodamine), diluted 1:200 in TBS, were incubated at $37^{\circ} \mathrm{C}$ for $30 \mathrm{~min}$. The cells were washed again $15 \mathrm{~min}$ in TBS, and the coverslips were mounted on glass slides with Glycergel (Dako Diagnostics, Zug, Switzerland).

Double labeling was performed combining each time a monoclonal antibody with the rabbit-anti-F protein antibody, mixing the primary antibodies in the same final concentrations as mentioned above. The secondary antibodies (goat anti-mouse-FITC, goat anti-rabbit-rhodamine) were also mixed in the same concentrations as above in TBS. All cultures were examined by confocal laser microscopy (LSM IV, Zeiss). All experiments were performed in duplicate.

\section{Virus titration}

To determine the amount of infectious CDV particles contained in the culture medium of the Vero cells, the supernatant fluids were titrated as previously described [23]. Briefly, supernatant fluids of Vero cells infected with OP-CDV or A75/17-CDV were collected $24,36,48$, and $72 \mathrm{~h}$ p.i. The supernatant fluids were then titrated on Vero cells. The amount of virus foci was monitored by immunostaining with the antibody against $\mathrm{N}$ protein, at $24 \mathrm{~h}$ p.i. of the target cells.

Biochemical analysis of the $\mathrm{F}$ protein

PCR amplified F genes of A75/17-CDV and OP-CDV were cloned into the plasmid pHGS-1, which contains a strong vaccinia virus late promoter [5]. These F proteins of A75/17 and OP-CDV were expressed using the vaccinia virus transient expression system [5]. Briefly, CV1 cells grown in 3.5-cm dishes were infected with vaccinia virus strain Western Reserve (WR) at an MOI of 5 in $400 \mu \mathrm{l}$ medium and left for $1 \mathrm{~h}$ at room temperature. Then, $2 \mathrm{ml}$ of medium were added and the cells were incubated for another $1 \mathrm{~h}$ at $37^{\circ} \mathrm{C}$. The cells were then transfected with $2.5 \mu \mathrm{g}$ vaccinia virus expression plasmids using the SuperFect reagent (Qiagen). Medium was changed $3 \mathrm{~h}$ after transfection and the cells were incubated for $15 \mathrm{~h}$ at $37^{\circ} \mathrm{C}$. The cells were then lysed in a buffer containing $20 \%$ glycerol, $2 \%$ SDS, $0.2 \%$ bromophenol blue, $100 \mathrm{mM}$ TRIS-HCl, pH6.8, and 5\% 2-mercaptoethanol. These samples were fractionated on $8 \%$ SDS-polyacrylamide gels under denaturing conditions and transferred to nitrocellulose membranes by electroblotting. The membranes were then soaked in TBS-Tween (25 mM TRIS- $\mathrm{HCl} \mathrm{pH} 7.5,137 \mathrm{mM} \mathrm{NaCl}, 3 \mathrm{mM} \mathrm{KCl}, 0.1 \%$ Tween 20) containing 5\% non-fat dry milk, incubated with the polyclonal rabbit antisera against the $\mathrm{F}$ protein at a dilution of 1:500. After one wash for $20 \mathrm{~min}$ and two washes for $5 \mathrm{~min}$ with TBS-Tween, the membranes were incubated for $1 \mathrm{~h}$ at room temperature with a 1:3,000 dilution in blocking buffer of a rabbit anti IgG antibody conjugated with horseradish peroxidase (Sigma). After three washes with TBS-Tween, the bound antibodies were detected with the enhanced chemiluminescence kit (Amersham) according to the manufacturer's instructions.

\section{Electron microscopy}

For ultrastructural studies, selected uninfected and infected Vero cell cultures were harvested at $48 \mathrm{~h}$ p.i. for the A75/17-CDV-in- 
Fig. 1 Morphological findings in cell cultures. a Infection of Vero cells with A75-CDV $48 \mathrm{~h}$ p.i. Heterogeneous pattern of infection with single scattered or small clumps of CDV-containing cells. Lack of cell fusion and cytolysis. Anti-N protein of CDV-PAP; DIC. b Infection of Vero cells with OP-CDV $24 \mathrm{~h}$ p.i. Compact pattern of infection with centrifugal spread to immediately adjacent cells, extensive cell fusion and central cytolysis of large syncytium. Anti-N of CDV-PAP; DIC. c Infection of Vero cells with A75-CDV and subsequent treatment with trypsin, at $48 \mathrm{~h}$ p.i. Formation of a large multinucleated syncytium. Anti-N of CDV-PAP; DIC ( $C D V$ canine distemper virus, p.i. post infection, $N$ nucleocapsid, DIC Differential interference contrast, $P A P$ peroxidaseanti-peroxidase, $O P$ Onderstepoort). $\mathbf{a}-\mathbf{c} \times 250$

fected cells, and $36 \mathrm{~h}$ p.i. for the OP-CDV-infected cells. The cells were fixed with $2.5 \%$ glutaraldehyde in $0.1 \mathrm{M}$ sodium cacodylate buffer for $1 \mathrm{~h}$ at room temperature. The cells were then osmificated $\left(1 \% \mathrm{OsO}_{4}\right.$ in $0.1 \mathrm{M}$ sodium cacodylate buffer) for $30 \mathrm{~min}$. The cell layer was detached with a rubber policeman and centrifuged for $5 \mathrm{~min}$ at $200 \mathrm{~g}$ in $0.1 \mathrm{M}$ cacodylate buffer. The cells were resuspended in $0.1 \mathrm{M}$ cacodylate buffer containing $10 \%$ bovine serum albumin (BSA). They were then pelleted by denaturing the BSA to a gel in a $25 \%$ glutaraldehyde solution. The pellets were dehydrated in graded ethanols and embedded in Texas (Epon-AralditeMix: Araldite MCY 212, Epon 812, Catalys, Serva). Ultrathin sections were stained using Reynold's method (double staining with lead citrate and uranyl acetate), and examined with a Zeiss 101 electron microscope.

\section{Results}

Morphological findings in infected cell cultures

In infected monolayers, A75/17-CDV, the persistent strain, initially produced foci consisting of single infected cells. The infection spread in a loose, net-ike pattern with single infected cells or small cell clusters separated by large numbers of uninfected cells (Fig. 1a). Infected cells remained intact. At $24 \mathrm{~h}$ p.i. some very rare small syncytia were observed. At $72 \mathrm{~h}$ p.i., the virus-containing foci had grown in size and eventually merged throughout the culture resulting in a confluent infection. The monolayer remained intact.

OP-CDV, the cytolytic strain, also initially produced a multifocal infection, but, in contrast to the persistent strain, the infection spread in a concentric manner forming large compact clumps of infected cells (Fig. 1b). Such foci formed syncytia which appeared from $18 \mathrm{~h}$ p.i. on. The syncytia reached considerable size with hundreds of nuclei which migrated centrally. Large syncytia became cytolytic with centrifugal destruction of the monolayer. At $48 \mathrm{~h}$ p.i., the culture was widely infected, and consisted mainly of large lytic syncytia. At $72 \mathrm{~h}$ p.i., the monolayer was largely destroyed.

Localization of the structural viral proteins by confocal laser microscopy

No major differences between the two strains were seen in the appearance and distribution of the major structural proteins in single-fluorescent-labeled preparations. Accu-
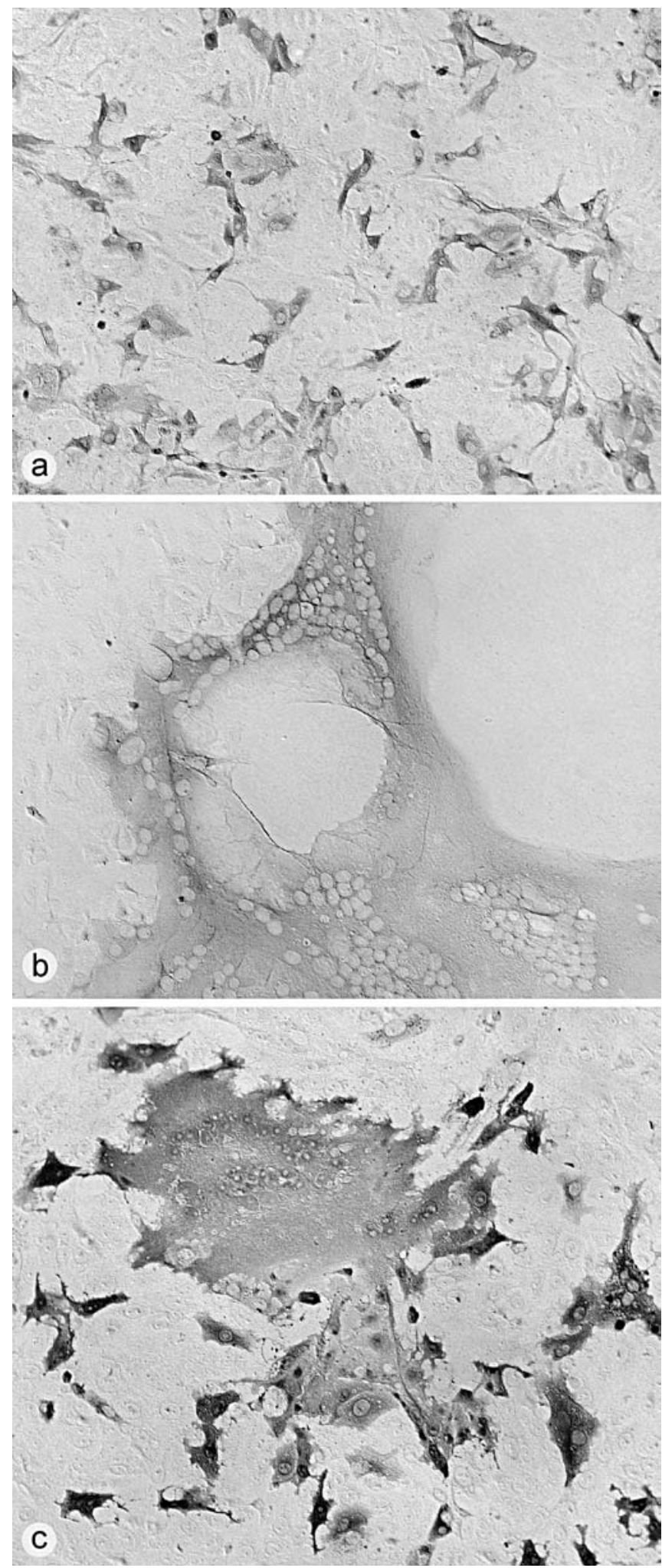

mulations of the surface proteins tended to be larger in the persistent infection. Double-immunofluorescence-labeling studies focused on intracellular granular aggregates or areas of higher focal concentrations of viral proteins in infected cells. In the case of the $\mathrm{N}$ protein such concentra- 
A75/17 - CDV

a

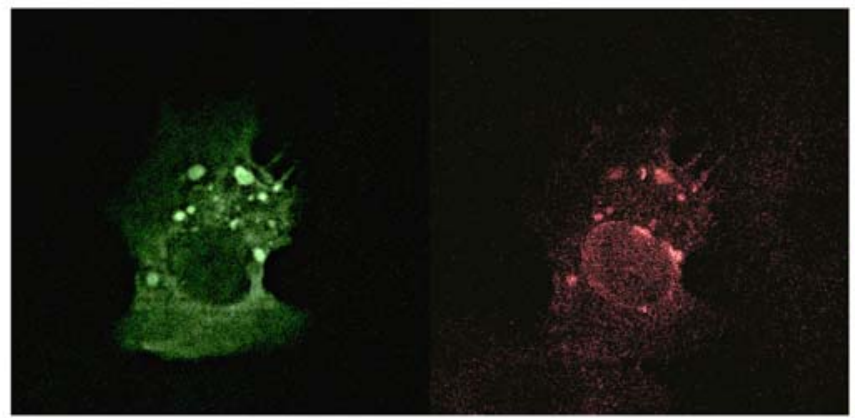

b
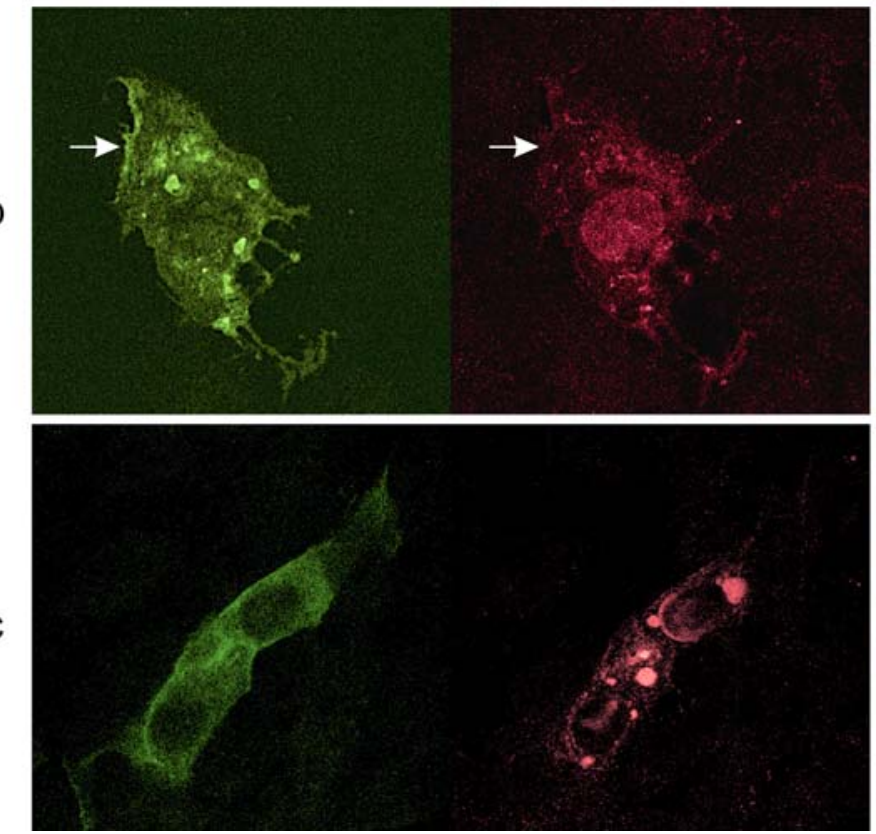
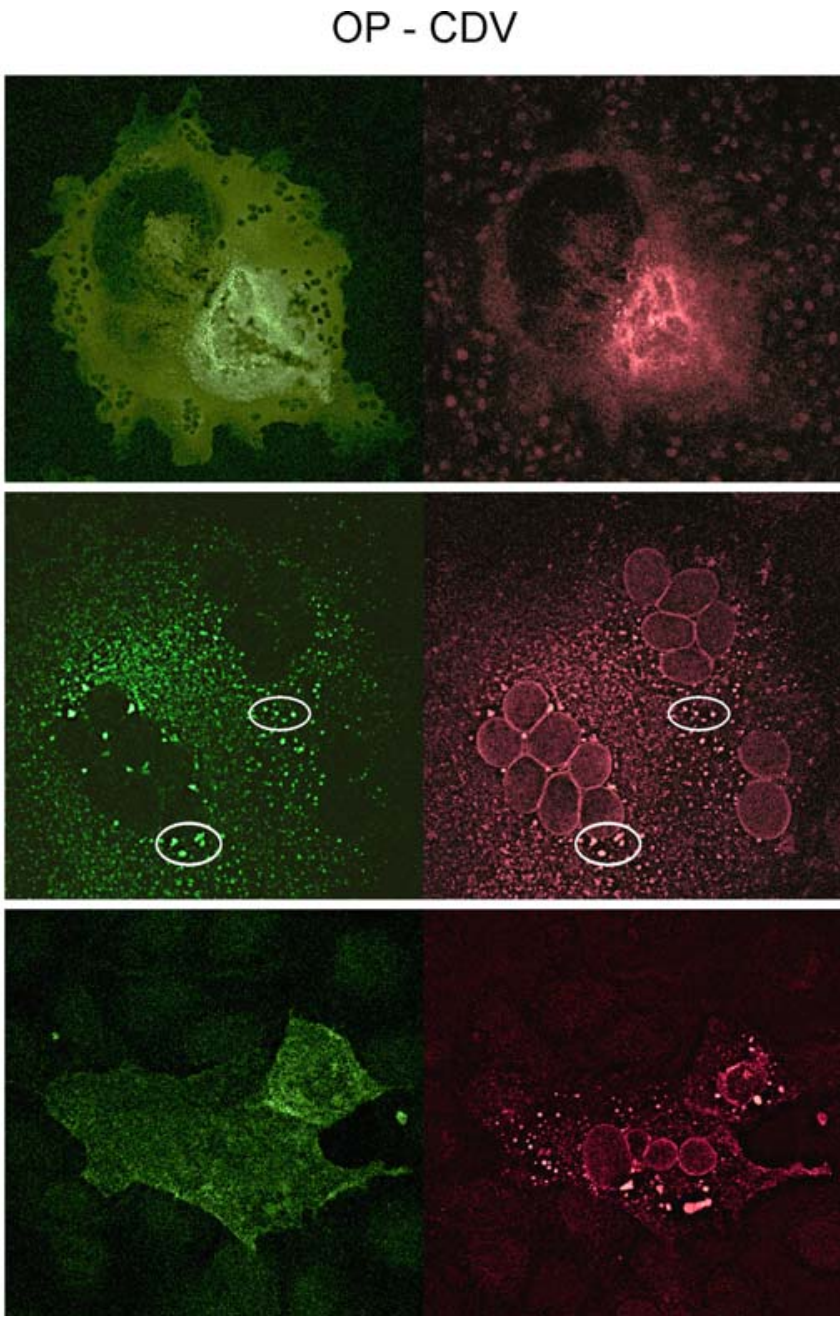

Fig. 2 Confocal laser microscopy. Double-labeling experiments using indirect immunofluorescence with either mouse antibodies against N, M, or H proteins detected by FITC (green) versus rabbit antibodies against $\mathrm{H}$ or $\mathrm{F}$ protein detected by rhodamine (red). Left: A75/17-CDV, right: OP-CDV. Evaluation focuses on antigen concentrations in patches. a Double labeling for $\mathrm{N}$ (green) and $\mathrm{H}$ (red). In A75/17-CDV some overlap of patches. In OP-CDV the largest concentration of both labels coincides with the highest concentration of virus. b Double labeling for $\mathrm{H}$ (green) and $\mathrm{F}$ (red). In A75/17-CDV, hardly any overlap between the two labels is seen. Patches do not match; stretch of cell membrane (arrow) is strongly labeled in $\mathrm{H}$ but not in $\mathrm{F}$ fluorescence. In OP-CDV there is complete overlap between $\mathrm{H}$ and $\mathrm{F}$ fluorescence (e.g., in two encircled areas). c Double labeling for M (green) and F (red). M is mostly present as finely granular evenly distributed fluorescence. No overlap with $\mathrm{F}$ patches in any of both infections is seen

tions reflect nucleocapsid aggregates in the cytosol, whereas accumulations of the surface glycoproteins are located in the endoplasmic reticulum.

By comparing the distribution of the $\mathrm{N}$ protein in respect to either the $\mathrm{F}$ protein or $\mathrm{H}$ protein, limited colocalization was observed in the OP-CDV- and A75/17-CDVinfected Vero cells (Fig. 2a). The distribution of the M protein in respect to the $\mathrm{F}$ or $\mathrm{H}$ protein showed that they were hardly colocalized in either infection (Fig. 2c). When comparing the distribution of the $\mathrm{H}$ in respect to the $\mathrm{F}$ pro- tein in OP-CDV infected cells, a striking colocalization was observed (Fig. 2b); exactly the same granular aggregates contained the $\mathrm{F}$ and the $\mathrm{H}$ protein. However, much less colocalization of the $\mathrm{F}$ and $\mathrm{H}$ proteins was observed in A75/17-CDV-infected cells.

Virus release in infected cultures

Vero cell cultures were infected using viral suspensions which had been diluted in such a way as to obtain a similar density of infected cells as judged following immunostaining after the initial $24 \mathrm{~h}$ p.i.

The results are summarized in Fig. 3. Both virus strains released infectious virus into the supernatant. A75/17-CDV could be detected in the supernatant fluid starting at $36 \mathrm{~h}$ p.i. A75/17-CDV titers increased up to $72 \mathrm{~h}$ p.i. OP-CDV was detectable in the culture medium already at $24 \mathrm{~h}$ p.i. with titers increasing up to $72 \mathrm{~h}$ p.i. The titers of the cytolytic infection were significantly higher (about a hundred fold) than those of A75/17-CDV at 24 and $48 \mathrm{~h}$. At the last sampling date, titers tended to converge because the cell layer in the cytolytic infection was largely destroyed. 
Fig. 3 Amount of infectious virus in supernatants derived from both infection types. Both virus strains released infectious virus into the supernatant. A75/17-CDV could be detected in the supernatant fluid starting at $36 \mathrm{~h}$ p.i. OP-CDV was detectable in the culture medium already at $24 \mathrm{~h}$ p.i. The levels of OP-CDV released were significantly higher (about a hundred fold) than those of A75/17-CDV at each time point. At $72 \mathrm{~h}$ p.i. titers tend to converge because the monolayer was largely destroyed in the cytolytic infection

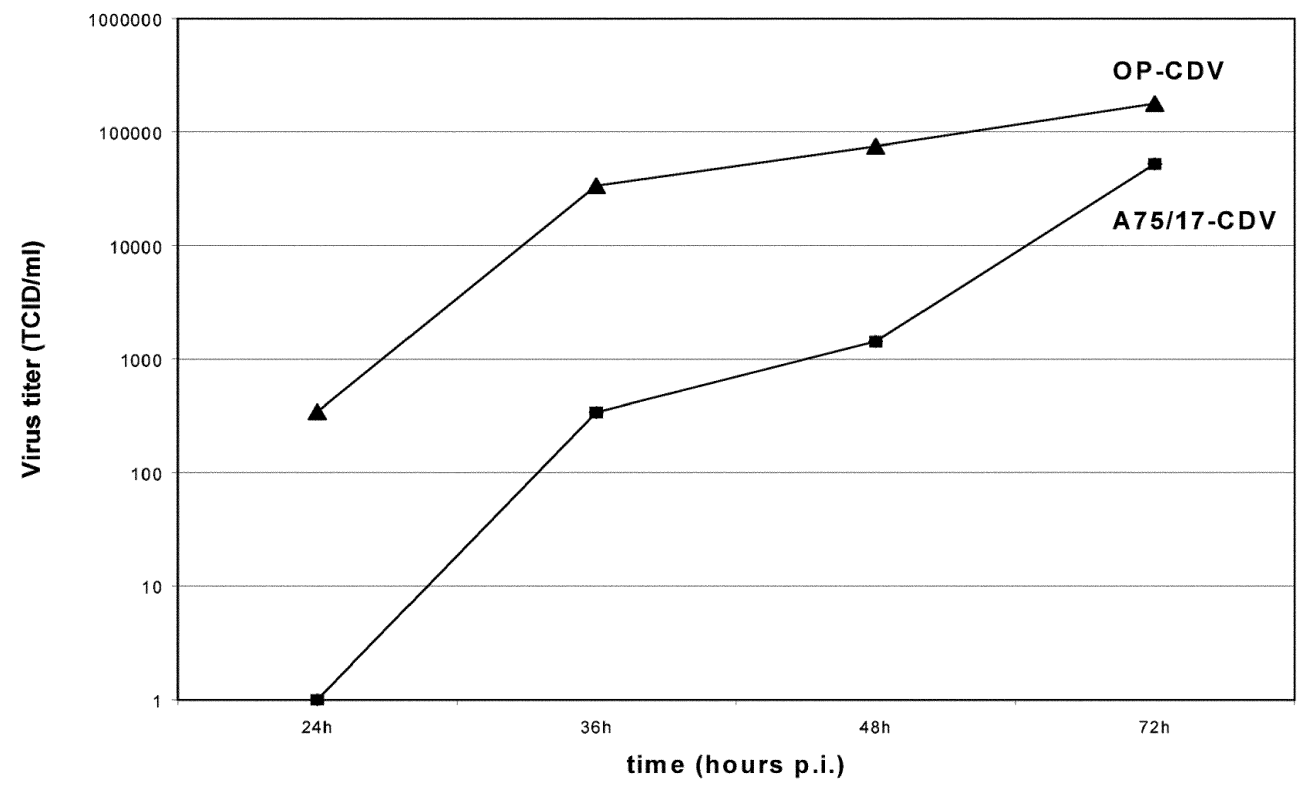

Transmission electron microscopy studies

In all infected cell cultures, virus was found as intracytoplasmic nucleocapsids and their aggregates, "spiking" of glycoproteins at the cell membrane, and budding particles at the cell surface. However, the budding activity of A75/ 17-CDV-infected cells was strongly reduced compared to that of the OP-CDV-infected cells. In the latter, extensive spiking was always associated with very intense shedding of viral particles at the cell surface (Fig. 4a, b). In the A75/17-CDV infection, spiking was seen over long stretches of the plasmalemmal membrane but was much less dense (Fig. 4c, d). Only relatively few viral buds were formed. Electron microscopy also confirmed the paucity of cell fusion in the A75/17-CDV infection.

Expression of A75/17 and OP-CDV F proteins using vaccinia constructs

Translation and subsequent cleavage of the inactive F0 protein precursors PreF0* and PreF0** and their cleavage products were examined by Western immunoblotting (Fig. 5) of Vero cells following transfection with vaccinia virus containing CDV F proteins of either strain. In extracts of cells expressing the A75/17 F and the OP-CDV F genes, a series of high molecular mass bands representing F0 precursors translated from two 2 different AUGs of the F gene were observed [5]. In addition, a band of about $46 \mathrm{kDa}$, the F1 cleavage product, the active fusion compound, was also detected by the antiserum.

The banding pattern of the high molecular mass precursors in cells expressing the OP-CDV gene was different from that obtained with strain A75/17-CDV. Particularly the two highest molecular mass species, PreF0* and $\mathrm{PreF0}^{* *}$, represented as intense bands with $\mathrm{A} 75 / 17$, were not visible with OP-CDV. These two proteins PreF0* and $\mathrm{PreF0}^{* *}$ are cleaved right after the signal peptide (between the amino acids 135 and 136, according to the numbering starting at the first $\mathrm{AUG}$ ) in the endoplasmic reticulum, resulting in the precursor F0. F0 is then cleaved during the transport in the Golgi in F1 and F2, the biologically active fusion protein. This observation and our previous site-directed mutagenesis [5] demonstrate that the OP-CDV PreF0s are more efficiently cleaved into the F0 precursor than A75/17.

Trypsin treatment of infected cells

Selected A75/17-CDV-infected Vero cell cultures were treated with trypsin. This resulted in the formation of few (four to eight syncytia per coverslip) but very large syncytia in all tested A75/17-CDV-infected cultures (Fig. 1c). No such syncytia were observed in the uninfected, trypsin-treated and in the infected, but not trypsin-treated cells.

\section{Discussion}

The mechanism of persistence of CDV, the driving force behind the progression of a debilitating demyelinating disease is not well understood. Our previous studies in primary brain cell cultures showed that persistence is associated with non-cytolytic cell-to-cell spread and limited production of virulent CDV [23]. It has been proposed that cell-to-cell viral spread in persistent infection with $\mathrm{MV}$, which is closely related to $\mathrm{CDV}$, could happen through microfusion between cells or cell processes and subsequent transport of nucleocapsids from one cell to the other $[7,12,15]$ or by transmission through the synapse in the case of neurons [10]. While observations in persistent CDV infection were suggestive of such mechanisms [23], concrete evidence of such unconventional cell-to-cell transmission has not been shown. 

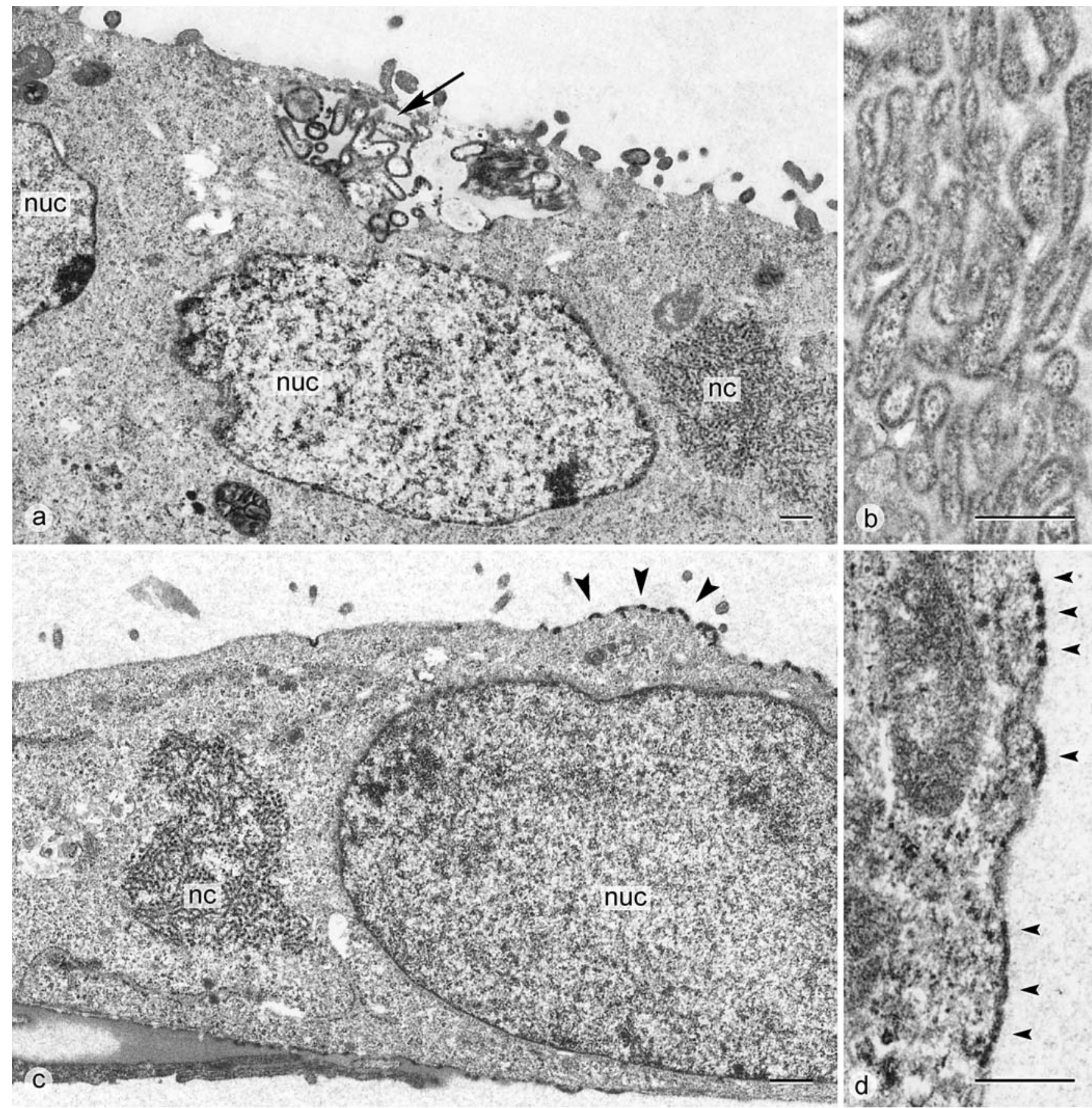

Fig. 4 Ultrastructural findings in infected Vero cells. a OP-CDV 24 h p.i. Part of a syncytium: two nuclei (nuc) are visible. Nucleocapsid $(n c)$ aggregates in the cytoplasm. One area of the cell membrane with profuse budding (arrow) can be seen. b OP-CDV $24 \mathrm{~h}$ p.i. At surface of a multinucleated syncytium, with production of large amounts of viral particles. c A75/17-CDV 36h p.i. Nucleocapsid $(n c)$ aggregates in cytoplasm. Glycoprotein spiking (arrowheads) in the cell membrane without budding is seen. d A75/17 CDV. Higher magnification shows glycoprotein spiking (arrowheads) in cell membrane. Bar $0.5 \mu \mathrm{m}$

In the present study, we investigated this question by comparing an attenuated non-persistent strain (OP-CDV) to a persistent CDV virus (A75/17-CDV) in Vero cells. The non-persistent virus spread concentrically, resulting in a compact pattern of infection with formation of large cytolytic syncytia. Immunostains of persistent CDV showed large numbers of uninfected cells between infected ones leading to a loose, net-like, heterogeneous pattern of infection. In contrast to OP-CDV-infected cells, cells infected with the persistent virus remained intact. The marked reduction of infectious titers in persistent infection, found in our titration experiments, correlated with our ultrastructural findings: relatively few viral buds were observed in persistently infected cells even though large amounts of nucleocapsids and spiking of glycoproteins were readily seen. In the present CDV model cell-to-cell spread can be 


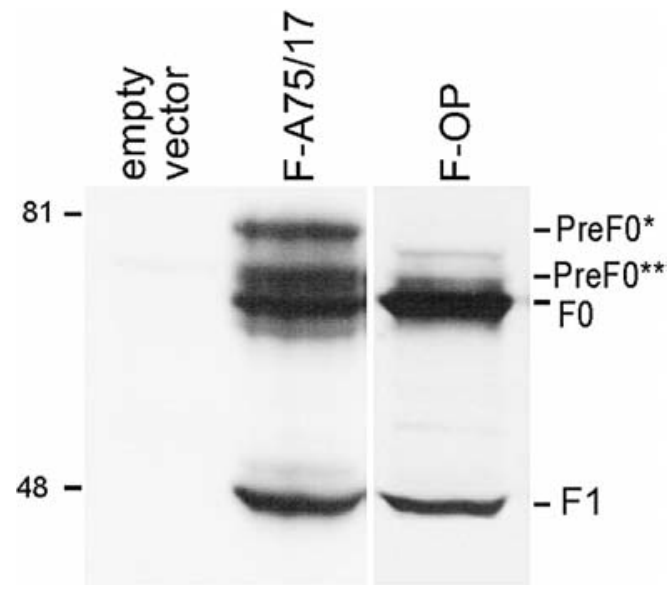

Fig. 5 Expression of A75/17-CDV and OP-CDV F proteins: Western blot analysis of cells transfected with empty vector and plasmids containing the $\mathrm{F}$ genes of A75/17-CDV or OP-CDV. The positions of the F0 and the F1 protein are indicated. In A75/17-CDV two additional higher molecular mass bands are seen corresponding to the two precursor $\mathrm{F}$ proteins ( $\mathrm{PreFo}^{*}$ and $\mathrm{PreFo}^{* *}$ )

simply explained by limited infectious viral particle production in only few areas of the cell membrane. In this way only a limited amount of surrounding cells becomes infected, resulting in a pattern of heterogeneous cell-to-cell spread typical for persistent morbilliviruses. Such pattern contrasts sharply with the compact concentric spread of profusely budding cytolytic virus. In CNS cells, which possess long cell processes, such heterogeneous spread is even more accentuated, as we have shown previously [23].

In addition to limited viral budding, persistence was also characterized by paucity of cell-cell fusion and lack of cytolysis as seen on light and electron microscopy. In MV infection, a causal relationship between the preF protein, cell-cell fusion and cytolysis has been established [6, 19]. Apparently, while not completely understood, the fusion process itself induces cell destruction as soon as a critical number of cells becomes involved in the formation of syncytia. Thus, lack of cytolysis of persistently infected cells appears to be determined by paucity of cell-cell fusion.

Clearly, lack of cell-cell fusion in persistent CDV infection must be related to a reduced expression of fusogenic complexes at the cell membrane. The latter consist of synergistically combined $\mathrm{F}$ and $\mathrm{H}$ proteins of $\mathrm{CDV}$, which are necessary for infectivity (viral attachment and penetration) [18] and fusion of adjacent cell membranes. Ultrastructurally, spiking of the glycoproteins was readily observed in both cytolytic and persistent infection but with much higher density in the former. Expression of the glycoproteins at the cell surface is required for successful budding, which involves complex interactions between all viral proteins $[3,14]$. Thus, in addition to lack of fusion, limited budding in persistent infection can also be related to reduced expression of glycoprotein complexes on the cell surface.

The evident paucity of fusogenic complexes in persistent infection could be linked to several other findings in the present study: the observed much lower degree of colocalization of the $\mathrm{F}$ and $\mathrm{H}$ proteins in A75/17-CDV than in OP-CDV by confocal laser microscopy suggests a decreased interaction between these glycoproteins, which is necessary for efficient fusion [21]. Furthermore, expression of $\mathrm{F}$ proteins of both $\mathrm{CDV}$ strains using vaccinia constructs in the present study showed that both F0 biotypes are cleaved to the active F1. However, cleavage of the F precursor proteins $\mathrm{PreFO}^{*}$ and $\mathrm{PreF0}{ }^{* *}$ (Cherpillod et al., submitted) into $\mathrm{F} 0$ in the endoplasmic reticulum is much less efficient in A75/17-CDV, than in OP-CDV. This may influence further processing and transport of the F proteins. A somewhat analogous situation was described in a SSPE MV strain in which F0 cleavage was markedly delayed [20] as compared to cytolytic MV. Since both F0 and $\mathrm{F} 1$ are inserted in the cell membrane in morbilliviruses [21], the relation of cleaved to uncleaved fusion protein could be in favor of the latter in A75/17-CDV infection. This view is supported by our experiment inducing large, albeit few, syncytia in A75/17-CDV infection following trypsin treatment.

In conclusion, lack of destruction of the cells supporting CDV infection as well as limited budding with a heterogeneous pattern of spread prevent and/or delay the activation of immune recognition mechanisms in the CNS, favoring persistence of the virus. The common denominator for these two characteristic features of CDV persistence, lack of cytolysis and cell-to-cell spread, is limited expression of active fusion complexes at the cell membrane. This, in turn, appears to result from restricted intracellular processing of the viral $\mathrm{F}$ protein. Previously reported molecular differences of the surface proteins between the two studied CDV strains [5] could explain such differences in intracellular processing. In the present system, persistence is determined by viral factors but could be further restricted by cell-specific factors, which have been shown to play a role in MV $[9,17]$.

Acknowledgements Grant number: Swiss National Science Foundation (31-58657.99)

\section{References}

1. Appel MJG, Gillespie JH (1972) Canine distemper virus. In: Virology monographs. Springer, Vienna, New York, pp 1-96

2. Bollo E, Zurbriggen A, Vandevelde M, Fankhauser R (1986) Canine distemper virus clearance in chronic inflammatory demyelination. Acta Neuropathol (Berl) 72:69-73

3. Cathomen T, Mrkic B, Spehner D, Drillien R, Naef R, Pavlovic J, Aguzzi A, Billeter MA, Cattaneo R (1998) A matrix-less measles virus is infectious and elicits extensive cell fusion consequences for propagation in the brain. EMBO J 17:3899-3908

4. Cattaneo R, Rose JK (1993) Cell fusion by the envelope glycoproteins of persistent measles viruses which caused lethal human brain disease. J Virol 67:1493-1502

5. Cherpillod P, Beck K, Zurbriggen A, Wittek R (1999) Sequence analysis and expression of the attachment and fusion proteins of canine distemper virus wild-type strain A75/17. J Virol 73:2263-2269 
6. Duprex WP, McQuaid S, Hangartner L, Billeter MA, Rima BK (1999) Observation of measles virus cell-to-cell spread in astrocytoma cells by using a green fluorescent protein-expressing recombinant virus. J Virol 73:9568-9575

7. Firsching R, Buchholz CJ, Schneider U, Cattaneo R, Meulen V ter, Schneider-Schaulies J (1999) Measles virus spread by cellcell contacts: uncoupling of contact-mediated receptor (CD46) downregulation from virus uptake. J Virol 73:5265-5273

8. Hamburger D, Griot C, Zurbriggen A, Örvell C, Vandevelde M (1991) Loss of virulence of canine distemper virus is associated with a structural change recognized by a monoclonal antibody. Experientia 47:842-845

9. Johnston IC, Meulen V ter, Schneider-Schaulies J, SchneiderSchaulies S (1999) A recombinant measles vaccine virus expressing wild-type glycoproteins: consequences for viral spread and cell tropism. J Virol 73:6903-6915

10. Lawrence DM, Patterson CE, Gales TL, D'Orazio JL, Vaughn MM, Rall GF (2000) Measles virus spread between neurons requires cell contact but not CD46 expression, syncytium formation, or extracellular virus production. J Virol 74:1908-1918

11. McQuaid S, Cosby SL (2002) An immunohistochemical study of the distribution of the measles virus receptors, CD46 and SLAM, in normal human tissues and subacute sclerosing panencephalitis. Lab Invest 82:403-409

12. McQuaid S, Campbell S, Wallace IJ, Kirk J, Cosby SL (1998) Measles virus infection and replication in undifferentiated and differentiated human neuronal cells in culture. J Virol 72: $5245-5250$

13. Müller CF, Fatzer RS, Beck K, Vandevelde M, Zurbriggen A (1995) Studies on canine distemper virus persistence in the central nervous system. Acta Neuropathol 89:438-445

14. Peeples ME (1991) Paramyxovirus M proteins: pulling it all together and taking it on the road. In: Kingsbury DW (ed) The paramyxoviruses. Plenum Press, New York, pp 427-456
15. Plumb J, Duprex WP, Cameron CH, Richter-Landsberg C, Talbot P, McQuaid S. (2002) Infection of human oligodendroglioma cells by a recombinant measles virus expressing enhanced green fluorescent protein. J Neurovirol 8:24-34

16. Schmid A, Spielhofer P, Cattaneo R, Baczko K, Meulen V ter, Billeter MA (1992) Subacute sclerosing panencephalitis is typically characterized by alterations in the fusion protein cytoplasmic domain of the persisting measles virus. Virology 188 : 910-915

17. Schneider-Schaulies J, Niewiesk S, Schneider-Schaulies S, Meulen V ter (1999) Measles virus in the CNS: the role of viral and host factors for the establishment and maintenance of a persistent infection. J Neurovirol 5:613-622

18. Stern LBL, Greenberg M, Gershoni JM, Rozenblatt S (1995) The hemagglutinin envelope protein of canine distemper virus (CDV) confers cell tropism as illustrated by CDV and measles virus complementation analysis. J Virol 69:1661-1668

19. Von Messling V, Cattaneo R (2002) Amino-terminal precursor sequence modulates canine distemper virus fusion protein function. J Virol 76:4172-4180

20. Watanabe M, Wang A, Sheng J, Gombart AF, Ayata M, Ueda S, Hirano A, Wong TC (1995) Delayed activation of altered fusion glycoprotein in a chronic measles virus variant that causes subacute sclerosing panencephalitis. J Neurovirol 1:412-423

21. Wild TF, Buckland R (1995) Functional aspects of envelopeassociated measles virus proteins. Curr Top Microbiol Immunol 191:51-64

22. Zurbriggen A, Yamawaki M, Vandevelde M (1993) Restricted canine distemper virus infection of oligodendrocytes. Lab Invest 68:277-284

23. Zurbriggen A, Graber HU, Wagner A, Vandevelde M (1995) Canine distemper virus persistence in the nervous system is associated with noncytolytic selective virus spread. J Virol 69: $1678-1686$ 